20. Wenzel RP. The emergence of methicillin-resistant Staphylococcus aureus. Ann Intern Med 1982;97:440-442.

21. Pérez Trallero E, Garcia Arenzana J, Ansa Castañeda A, Paisán Grisolía L. Unusual multiresistant Staphylococcus aureus in a newborn nursery. Am J Dis Child 1981;135:689-692.

22. Voss A, Milatovic D, Wallrauch-Schwarz C, Rosdahl VT, Braveny I. Methicillin-resistant Staphylococcus aureus in Europe. Eur I Clin Microbiol Infect Dis 1994;13:50-55.

23. Markowitz N, Pohlod DJ, Saravolatz LD, Quin EI. In vitro susceptibility patterns of methicillin-resistant and susceptible Staphylococcus aureus strains in a population of parenteral drug abusers from 1972 to 1981. Antimicrob Agents Chemother 1983;23:450-457.

24. Parras F, Guerrero C, Bouza E, et al. Comparative study of mupirocin and oral cotrimoxazole plus topical fusidic acid in eradication of nasal carriage of methicillin-resistant Staphylococcus aureus. Antimicrob Agents Chemother 1995;
$39 \cdot 175-179$

25. Hill RL, Duckworth GH, Casewell MW. Elimination of nasal carriage of methicillin-resistant Staphylococcus aureus with mupirocin during a hospital outbreak. J Antimicrob Agents Chemother 1988;22:377-384.

26. Samra Z, Gadba R. Antibiotic susceptibility and phage typing of methicillin-resistant Staphylococcus aureus clinical isolates from blood cultures of 692 patients in 15 Israeli hospitals. Eur J Epidemiol 1993;9:559-562.

27. Qadri SM, Halim M, Ueno Y, Saldin H. Susceptibility of methicillin-resistant Staphylococcus aureus to minocycline and other antimicrobials. Chemotherapy 1994;40:26-29.

28. Goetz MB, Mulligan ME, Kwork R, O'Brien H, Caballes C, García JP. Management and epidemiologic analysis of an outbreak due to methicillin-resistant Staphylococcus aureus. Am J Med 1992;6:607-614.

\title{
Microbiological Factors and Mortality of Septicemia
}

\section{Gina Pugliese, RN, MS Martin S. Favero, PhD}

Each year, approximately 250,000 of the 35 million patients admitted to hospitals in the United States develop nosocomial bloodstream infections. The age-adjusted death rate due to septicemia (nosocomial and community-acquired combined) has shown a progressive linear increase over the past 4 decades from 0.3 deaths per 100,000 population in 1950 to 7.9 per 100,000 population in 1993 . Studies have shown that mortality rates associated with nosocomial bloodstream infections are higher than those associated with community-acquired infections. The crude mortality associated with bloodstream infections is approximately $35 \%$, ranging from $12 \%$ to $80 \%$. The attributable mortality (mortality directly due to infection after controlling for underlying disease) averages $25 \%$ but varies with the organism causing the infection.

Investigators from the University of Iowa Hospitals and Clinics conducted a 6-year study to identify those microbiological factors that are associated independently with mortality due to nosocomial bloodstream infections. All patients admitted to the hospital who developed a nosocomial bloodstream infection between July 1 , 1986, and June 30, 1991, were includ- ed in the study. Patients were identified by prospective, case-based surveillance and positive blood cultures. Mortality rates were examined for secular trends. Prognostic factors were determined with the use of univariate and multivariate analyses, and both derivation and validation sets were used.

A total of 1,745 patients developed nosocomial bloodstream infection. The 28-day crude mortality was $22 \%$, and crude in-hospital mortality was $35 \%$. Factors independently (all $P<.05)$ associated with increased 28day mortality rates were older age, longer length of hospital stay before bloodstream infection, and a diagnosis of cancer or disease of the digestive system. After adjustment for major confounders, Candida species were the only organisms independently influencing the outcome of nosocomial bloodstream infection (odds ratio [OR] for mortality, 1.84; 95\% confidence interval $\left[\mathrm{CI}_{95}\right], 1.22-2.76$; $P=.0035)$. The two additional microbiological factors independently associated with increases in mortality were pneumonia as a source of secondary infection (OR, 2.74; $\mathrm{CI}_{95}, 1.87-4.00$; $P<.0014)$ and polymicrobial infection (OR, 1.68; $\left.\mathrm{CI}_{95}, 1.22-2.32 ; P=.0014\right)$.

The authors point out that, given the high mortality attributable to candidemia, single positive cultures should not be regarded as representing benign, transient colonization. The difficulty of predicting which patients will develop disseminated candidiasis and the low sensitivity of blood cultures in such conditions, coupled with the availability of systemic antifungals with lower toxicity than amphotericin $\mathrm{B}$, have led to the consensus that all cases of candidemia should be treated. Because the yield of blood cultures depends on the volume of blood cultures and because the sensitivity of low-volume blood cultures for yeast is particularly low, it is recommended that highvolume (at least $10 \mathrm{~mL}$ ) blood cultures be done for patients at high risk for candidemia.

The authors concluded that microbiological factors independently can affect the outcome of nosocomial bloodstream infection.

This is the largest published study to measure the direct impact of microbiological factors on the outcome of nosocomial bloodstream infections, after controlling for major confounders.

FROM: Pittet D, Li N, Woolson R, Wenzel R. Microbiological factors influencing the outcome of nosocomial bloodstream infections: a 6-year validated, population-based model. Clin Infect Dis 1997;24:1068-10678. 